\title{
Memory effect in weakly anchored surfaces of deformed helix ferroelectric liquid crystals
}

\author{
J. Prakash, ${ }^{1,2}$ A. Choudhary, ${ }^{1}$ S. Kaur, ${ }^{1}$ D. S. Mehta, ${ }^{2}$ and A. M. Biradar ${ }^{1, *}$ \\ ${ }^{1}$ National Physical Laboratory, Dr. K. S. Krishnan Road, New Delhi 110 012, India \\ ${ }^{2}$ Instrument Design and Development Center, Indian Institute of Technology Delhi, Hauz Khas, New Delhi-110016, India
}

(Received 4 March 2008; published 15 August 2008)

\begin{abstract}
A wide-range memory effect in deformed helix ferroelectric liquid crystals (DHFLCs) has been investigated by electro-optical and textural methods. A comparative study has been performed on strongly and weakly anchored surfaces of DHFLC cells. The saturation voltage has been compared in both types of cells by studying the variation of tilt angle and spontaneous polarization with applied voltage. The long-lasting memory effect has been observed in untreated (weakly anchored) cells. It has been proposed that perfect memory in DHFLC cells without any surface treatment is due to the possibility of the absence of depolarization and ionic charges over the surfaces of the cells.
\end{abstract}

DOI: 10.1103/PhysRevE.78.021707

PACS number(s): 77.84.Nh, 42.79.Kr, 83.80.Xz

\section{INTRODUCTION}

Numerous potential applications of liquid crystals (LCs) allows researchers to study different aspects of their basic properties, particularly the surface-anchoring effect, which forces near-surface molecules to align molecules in bulk in different modes-i.e., homogeneous or homeotropic. The surface alignment is essential in liquid crystal displays (LCDs) as it controls the director of one of the two states of the display. Almost all kinds of electro-optic devices such as nematic, twisted nematic (TN), super twisted nematic (STN), and the ferroelectric liquid crystal (FLC) are fabricated using a surface alignment technique. The LC materials are anchored at the substrate along the preferred direction induced by the surface. There are several alignment techniques such as rubbed polyimide films [1], oblique evaporation of $\mathrm{SiO}$ films [2], polyimide Langmuir-Blodgett films [3], and polystyrene films [4]. An alignment method for obtaining excellent surface-stabilized ferroelectric liquid crystals (SSFLCs) by obliquely depositing two orthogonal $\mathrm{SiO}$ layers has been demonstrated by Bawa et al. [5]. The surface plays a passive role in LCDs currently available on the market. But the situation becomes completely different when the surface plays an active role [6]. The surface-anchoring effect in nematic liquid crystals (NLCs) has been studied extensively for several decades to understand the precise interaction between the surface and LC materials [7,8]. But the surface-anchoring effect studies in FLCs are rather more difficult and rarely reported in the literature due to the complexity of surface interactions caused by the introduction of spontaneous polarization and layer structure.

There are two types of surface-anchoring energies [9], which define the anchoring effect in LCs. These are azimuthal and polar anchoring energies. The azimuthal anchoring energy is defined as the energy per unit area for a $90^{\circ}$ rotation in the substrate plane whereas polar (or zenithal) anchoring energy is defined as the energy per unit area required to rotate the near surface director by $90^{\circ}$ out of plane. Various methods have been reported to measure these two

\footnotetext{
*Corresponding author. abiradar@mail.nplindia.ernet.in
}

anchoring energies. The azimuthal anchoring energy can be measured by the optical method [10], torque balance method [11], magnetic field distortion method [12], and Cano wedge method [13]. On the other hand, the polar anchoring energy can be measured by the electrical method [14] using a twochannel scheme for capacitance measurement and by curve fitting of the capacity of the homogeneous cells versus applied voltage [15]. As far as the display devices are concerned, the surface director switches between the two states of the display. In order to achieve this requirement, weak anchoring becomes important. Another reason to take weak anchoring into account is that the well-known rubbed polymer alignment technique has several disadvantages. The main disadvantage is the possibility of deposition of dust particles on the surface that decrease the manufacture yield [16] and the other one is the possession of zigzag defects which affect the contrast of the display devices [17]. Various methods such as soft rubbing of polymers [18], photosensitive polymer layers in combination with polarized light [16,19-21], and addition of oligomeric molecules to LCs [9] have been presented to produce weak anchoring. The weak anchoring effect in NLCs has been studied well $[9,16]$. But such an effect in FLCs has not been studied. The effect of surface anchoring on switching dynamics of SSFLCs is also not well understood as the switching in these FLCs takes place in the plane of the bounding substrate [22]. However, Clark and Lagerwall explained few possible effects of anchoring in SSFLC [23]. Beresnev et al. [24] demonstrated the deformation of helix and called it the deformed helix ferroelectric (DHF) effect and subsequently this effect was used to fabricate fast-responding liquid crystal devices based on this effect [25]. The specialty with deformed helix ferroelectric liquid crystals (DHFLCs) material is that the gray level can be easily realized in these materials, as the pitch is almost linearly dependent on the electric field [26]. Apart from this, these materials have lot of advantages over SSFLCs such as better contrast and thickness-independent memory effect. Few studies such as switching dynamics [27], grayscale characteristics [17], and symmetric switching [28] have been performed in high-tilt-angle FLCs.

In this paper, an ultraweak anchoring effect has been studied in DHFLC material. Here we show how an ultraweak anchoring can be used to lower the saturation voltage. The 

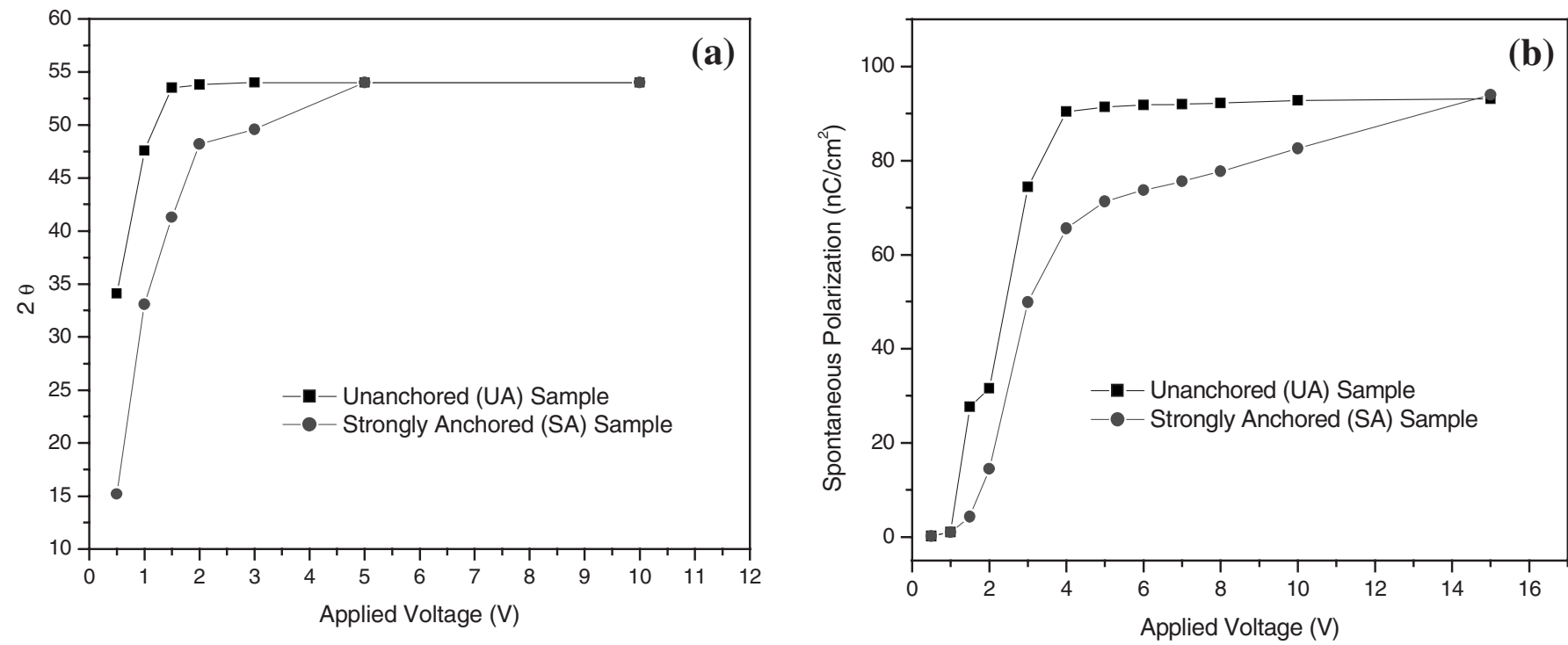

FIG. 1. (a) Behavior of the optical tilt $(2 \theta)$ as a function of bias voltage for SA and UA samples. (b) The variation of spontaneous polarization with applied voltage.

perfect memory effect in a broad frequency range in DHFLC material without any surface treatment is shown. However, the achievement of almost monodomain DHFLC cells and the reproducibility in untreated glass substrates were around $20 \%-40 \%$. The experimental data have been analyzed by electrical, optical, and textural methods in DHFLC cells.

\section{EXPERIMENT}

The sample cells for the present study were prepared using ITO glass plates. Three types of samples were prepared. In the first type, two electrodes were treated with polymer and strongly rubbed; we call them strongly anchored (SA) cells. In the second type, both electrodes were coated with polymer and then rubbed normally (or gently) and they are called medium anchored (MA) cells. In the third type, both electrodes were left untreated (i.e., the polymer was neither coated nor rubbed) and they are called unanchored (UA) cells. Rubbing in the case of the first and second types of samples (whether strongly or gently) of polymer-coated glass plates was done by using a buffing machine (LCBM, Irwin, Pennsylvania) to obtain homogeneous alignment. On the other hand, the possibility of planar alignment in the third type of samples was due to a concept given by Miyano [29] who described that LCs could have a rather stable planar structure while it has no preferential orientation-i.e., no surface anchoring.

The glass plates were assembled into cells maintaining a uniform gap of around $3.5 \mu \mathrm{m}$ using photoresist material at the periphery by means of the photolithographic technique. In this study, a DHFLC material (FLC 6304, Rolic, Allschwil, Switzerland) having spontaneous polarization of $90 \mathrm{nC} / \mathrm{cm}^{2}$ and a pitch value of $0.35 \mu \mathrm{m}$ was used. The phase sequence of this material is as follows:

$$
\begin{gathered}
-14^{\circ} \mathrm{C} \quad{ }^{60.5^{\circ} \mathrm{C}} \quad{ }^{6{ }^{\circ} \mathrm{C}} \\
\text { cryst. } \leftrightarrow \operatorname{Sm}^{*} \leftrightarrow \operatorname{Sm} A \leftrightarrow \text { iso. }
\end{gathered}
$$

The material was introduced into the cells by means of capillary action at elevated temperature to ensure that filling takes place in the isotropic phase. The smectic layers were arranged with layer planes perpendicular to the cell surfaces. The electro-optical measurements were carried out by applying an electric field parallel to the smectic layers. Saturation voltage and tilt angle measurements were carried out by observing the texture under a polarizing microscope (Ax-40, Carl Zeiss, Goettingen, Germany) on application of the bias field. The spontaneous polarization measurement was performed using an automatic liquid crystal tester (ALCT, Instec, Boulder, Colorado). The electrical response was observed by applying electrical pulses of triangular shape generated from a pulse generator to the sample. For optical response, the sample was mounted on a polarizing microscope and the transmission of normally incident polarized light through the sample and analyzer was monitored with a photodiode. The time delayed square pulse generated from the pulse generator was applied to the sample and studied by using a storage oscilloscope (HM 1507-3, HAMEG, Frankfurt, Germany) interfaced with the computer via SP-107 software. The textural studies were carried out by mounting the sample under a polarizing microscope attached with a computer-controlled charge-coupled-device camera.

\section{RESULTS AND DISCUSSIONS}

The weak anchoring has been found to lower the saturation voltage in LCs $[9,16]$. The saturation voltage has been compared in SA and UA sample cells. These studies could be inferred by taking the variation of tilt angle for different dc voltages, which is shown in Fig. 1(a). First the tilt angle increases at lower voltages. On further increasing the field the tilt angle starts to saturate and no change was observed beyond a certain voltage. But the saturation in tilt angle in the UA sample was found almost at the half dc voltage to that of the saturation for the SA sample. The saturation voltage $V_{s}$ is dependent on the anchoring energy as described earlier [30] in the case of nematic liquid crystals. The dependence can be shown as follows 


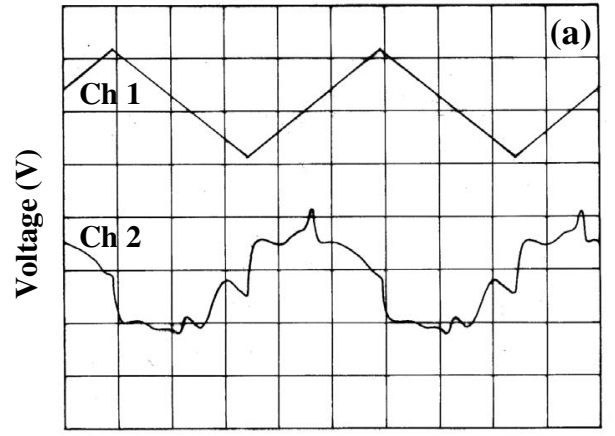

Time (s)

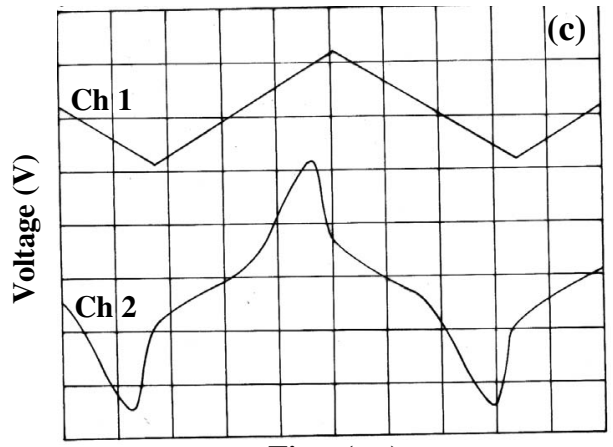

Time (ms)

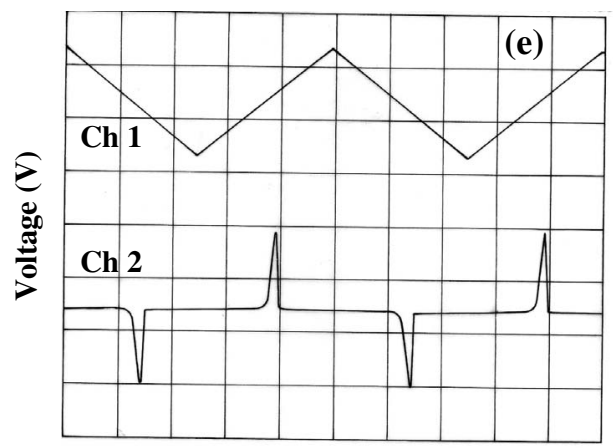

Time (ms)

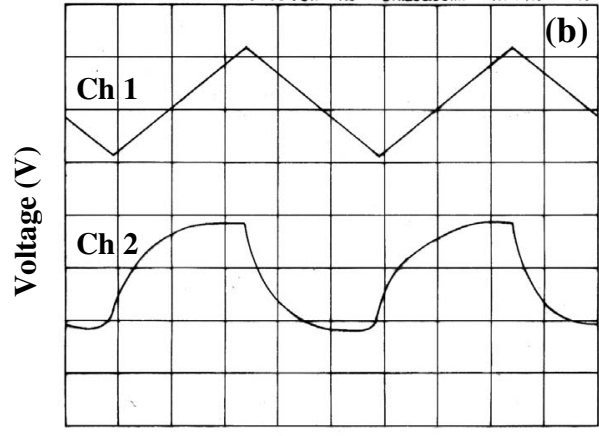

Time (ms)

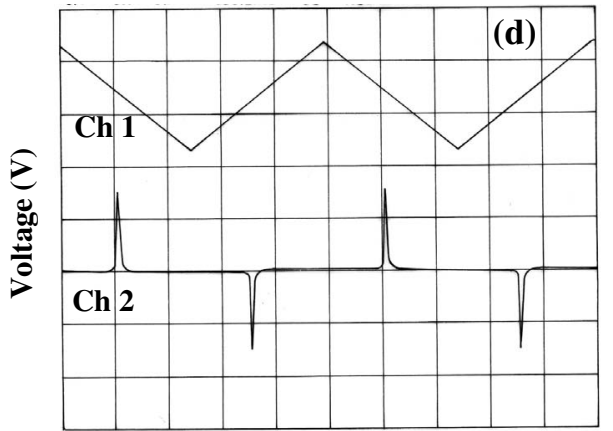

Time (s)

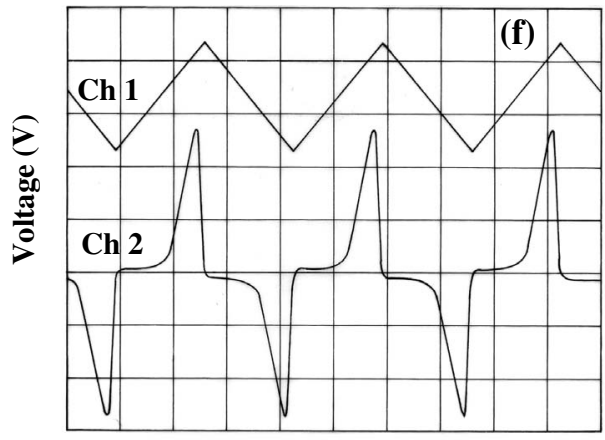

Time (ms)

FIG. 2. Electrical response of DHFLCs at room temperature in 3.5- $\mu \mathrm{m} \mathrm{SA} \mathrm{(a)-(c)} \mathrm{and} \mathrm{UA} \mathrm{(d)-(f)} \mathrm{sample} \mathrm{cells} \mathrm{at} 20 \mathrm{~V}$ at (a) $100 \mathrm{mHz}$ (Ch 1, $10 \mathrm{~V} / \mathrm{div}$; Ch 2, $0.05 \mathrm{~V} /$ div; time, $2 \mathrm{~s} / \mathrm{div})$, (b) $1 \mathrm{~Hz}(\mathrm{Ch} 1,10 \mathrm{~V} / \mathrm{div}$; Ch 2, $0.5 \mathrm{~V} / \mathrm{div}$; time, $200 \mathrm{~ms} / \mathrm{div})$, (c) $30 \mathrm{~Hz}(\mathrm{Ch} 1$, $10 \mathrm{~V} / \operatorname{div}$; Ch 2, $2 \mathrm{~V} / \mathrm{div}$; time, $5 \mathrm{~ms} / \mathrm{div})$, (d) $100 \mathrm{mHz}(\mathrm{Ch} 1,10 \mathrm{~V} / \mathrm{div} ; \mathrm{Ch} 2,1 \mathrm{~V} / \mathrm{div} ;$ time, $2 \mathrm{~s} / \mathrm{div}),(\mathrm{e}) 1 \mathrm{~Hz}(\mathrm{Ch} 1,10 \mathrm{~V} / \mathrm{div} ; \mathrm{Ch} 2$, $0.5 \mathrm{~V} /$ div; time, $200 \mathrm{~ms} / \mathrm{div})$, and (f) $30 \mathrm{~Hz}$ (Ch 1, $10 \mathrm{~V} / \mathrm{div}$; Ch 2, $2 \mathrm{~V} / \mathrm{div}$; time, $10 \mathrm{~ms} / \mathrm{div})$.

$$
W_{\phi}=\frac{V_{s} \sqrt{\varepsilon_{0} \Delta \varepsilon K}}{d},
$$

where $d$ is the cell gap, $\varepsilon_{0}$ is the free-space dielectric permittivity, $\Delta \varepsilon$ is the dielectric anisotropy, and $K$ is the average of the splay-and-bend elastic constant.

Using the same analogy, it has been shown that the saturation voltage for UA samples is almost half of the saturation voltage for SA samples because no surface treatment was given to UA samples. The lowering in switching (threshold) voltage can be correlated with the increase in spontaneous polarization in UA samples as shown in Fig. 1(b). The threshold voltage of DHFLC material is related to spontaneous polarization as follows:

$$
E_{t h} \propto \frac{K}{L^{2} P_{s}},
$$

where $E_{t h}$ is the threshold field, $K$ is the torsional elastic constant for the helix, $L$ is the pitch of the helix, and $P_{S}$ is the spontaneous polarization. One can clearly see from Fig. 1(b) that there is a remarkable difference in polarization values of SA and UA samples in the saturation region.

Figure 2 shows the electrical response of DHFLC material for SA and UA samples, respectively, for different frequencies at a fixed voltage on the application of triangular wave pulses. One can see from Figs. 2(a)-2(c) that no clear (symmetric) polarization peak, particularly at low frequencies (from $100 \mathrm{mHz}$ to $10 \mathrm{~Hz}$ ), was observed. There is a large nonlinearity in the output response of the SA samples. Such 


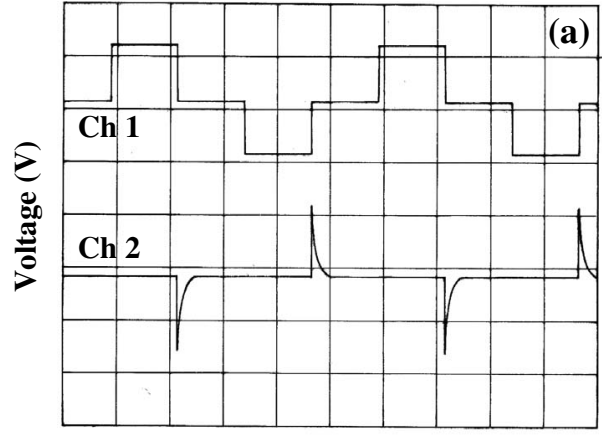

Time (s)

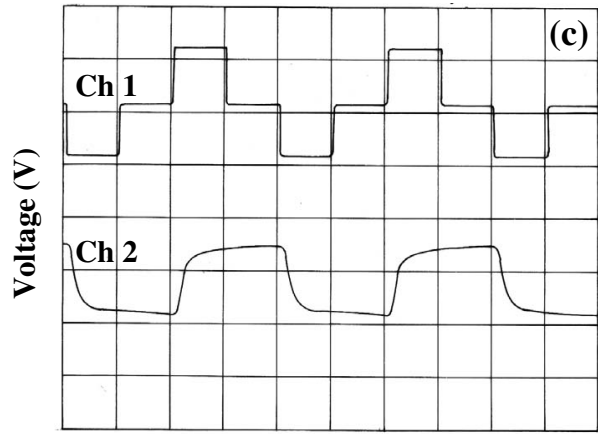

Time $(\mu \mathrm{s})$

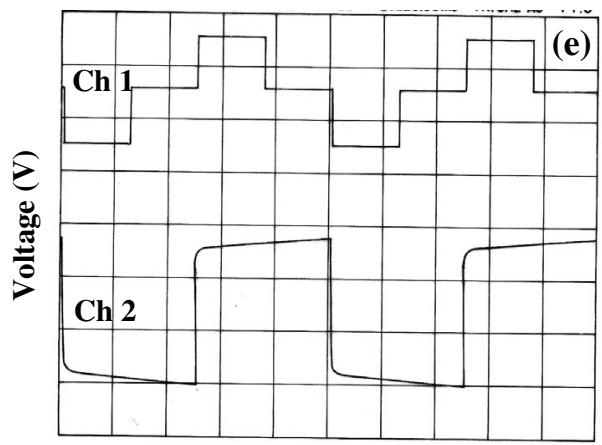

Time (ms)

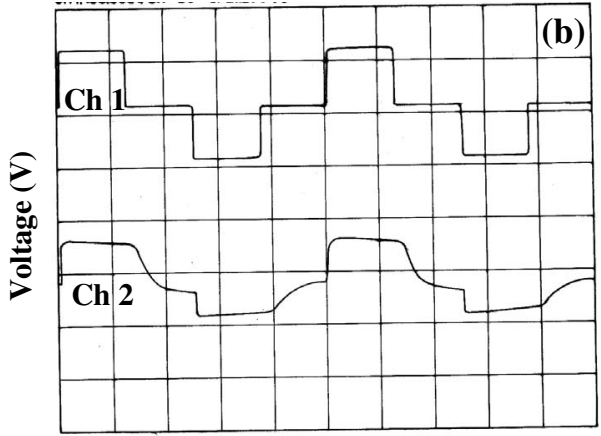

Time (ms)

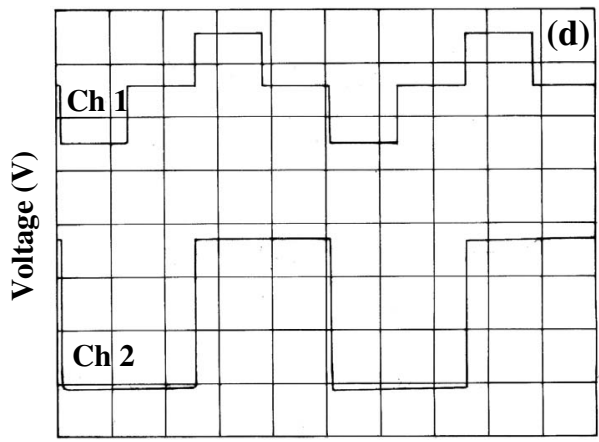

Time (s)

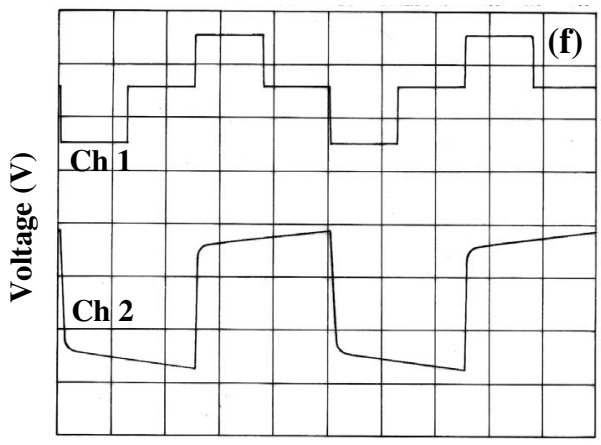

Time (ms)

FIG. 3. Optical response of DHFLCs at room temperature in 3.5- $\mu \mathrm{m} \mathrm{SA} \mathrm{(a)-(c)} \mathrm{and} \mathrm{UA} \mathrm{(d)-(f)} \mathrm{sample} \mathrm{cells} \mathrm{at} 20 \mathrm{~V}$ at (a) $100 \mathrm{mHz}$ (time, $2 \mathrm{~s} / \mathrm{div}$ ), (b) $10 \mathrm{~Hz}$ (time, $20 \mathrm{~ms} / \mathrm{div}$ ), (c) $100 \mathrm{~Hz}$ (time, $500 \mu \mathrm{s} / \mathrm{div}$ ), (d) $100 \mathrm{mHz}$ (time, $2 \mathrm{~s} / \mathrm{div}$ ), (e) $10 \mathrm{~Hz}$ (time, $20 \mathrm{~ms} / \mathrm{div}$ ), and (f) $100 \mathrm{~Hz}$ (time, $2 \mathrm{~ms} / \mathrm{div}$ ). The voltage on the $y$ axis for channels Ch 1 and Ch 2 are $10 \mathrm{~V} / \mathrm{div}$ and $0.02 \mathrm{~V} /$ div, respectively.

nonlinear behavior also has been reported for $10-\mu \mathrm{m}$ samples of p-decycloxybenzylidene- $p^{\prime}$-amino-2-methylbutyl cinnamate (DOBAMBC) $[31,32]$. This nonlinearity is caused by polarization charges at the interfaces between the FLC medium and the insulating alignment layers. These interfacial charges generate the depolarization field to reverse the direction of spontaneous polarization. The rate of this reverse switching is proportional to the product of the spontaneous polarization and depolarization field [33]. Second, it has been observed that if the pulse width of the external voltage is larger than approximately a few hundred $\mu$ s, then accumulation of excess ions at the interface between the FLC medium and the alignment layers takes place [34]. These excess ions create the external field, which adds to the depolarization field and becomes responsible for the nonlinearity in the electrical response of SA samples. On the other hand, a symmetric polarization peak was observed in UA samples in a broad frequency range (from $100 \mathrm{mHz}$ to $1 \mathrm{kHz}$ ), which is reflected clearly in Figs. 2(d)-2(f). The absence of alignment layers and their rubbing injects free charges to the interface, which screen or neutralize the interfacial charges, and causes the depolarization field and ionic charge effect to vanish. Similar observations of the polarization peak for studying the bistability in DHFLC material have been published elsewhere [35].

Figure 3 shows the optical response of DHFLC material for SA [Figs. 3(a)-3(c)] and UA [Figs. 3(d)-3(f)] samples, respectively, for different frequencies at a fixed voltage on the application of time-delayed square wave pulses. As one can see from Figs. 3(a)-3(c) there is no memory effect at low frequencies, whereas little memory effect was observed at higher frequencies. The memory effect in DHFLCs could be seen at a certain frequency and voltage as explained earlier [36]. On the other hand, a complete memory effect was ob- 

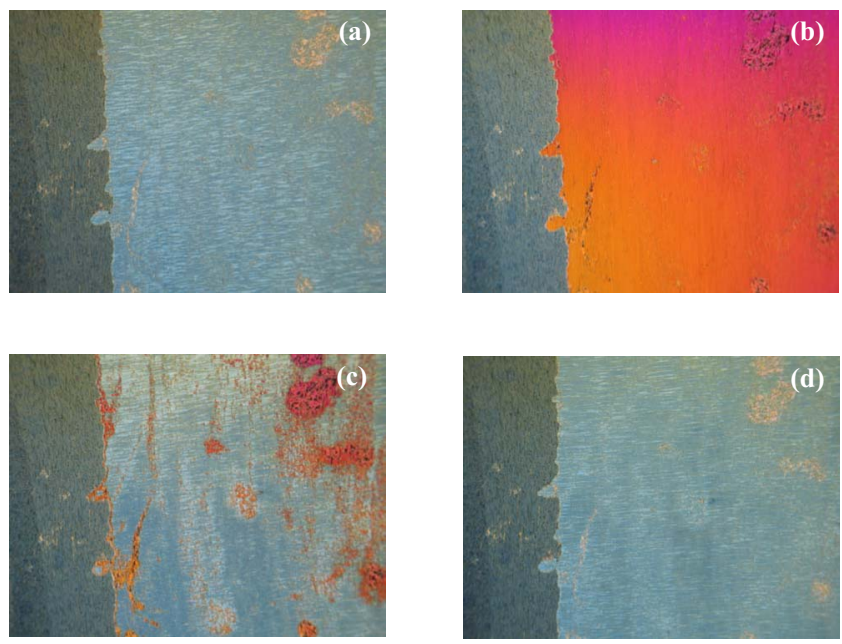

FIG. 4. (Color online) Optical micrographs of 3.5- $\mu \mathrm{m}$ SA cells at room temperature at (a) $0 \mathrm{~V}$, (b) $15 \mathrm{~V}$ bias, (c) $10 \mathrm{~min}$ after removal of bias, and (d) again $0 \mathrm{~V}$.

served in an entire frequency range in UA samples as shown in Figs. 3(d)-3(f). It was due to the fact that there was no alignment layer and no surface treatment to the cell; the conducting FLC medium was directly in contact with the conducting ITO coating. So there was an injection of free charges, which screen or neutralize the depolarization and ionic fields.

The complete memory effect in UA cells can also be correlated with the electrical response of the same cells. It has been reported that if there was single peak in electrical response, meaning perfect resonance between helix deformation and molecular reorientation processes, then a complete memory effect would be observed [35]. We found that the results are consistent with those observed earlier where the criticality of the bistability of DHFLCs was taken into account due to surface treatment of the cell substrates. The optical memory effect was also observed in MA samples. The memory effect in such samples was found in between the memory effect of UA and SA samples. It is worth mentioning here that minimum anchoring leads to a better memory effect ever observed in DHFLC materials It should be mentioned here that the bistable switching (memory effect) in other DHFLC (FLC 6430) materials, having different pitch values and phase transition temperatures, was studied earlier by Jakli et al. [37] where weak anchoring enabled helical director structure persisting at the surface resulting in the memory effect. The weak anchoring was obtained in their case by using silane-treated surfaces, but in the present study no surface treatment was given. Second, the memory effect shown earlier was not long lasting, but a transient one. The present study shows the memory effect due to stabilization of helix deformation and can be static and stays in a stable (memory) state for longer duration (hours).

The memory found in UA samples was confirmed by observing the texture in a deep $\mathrm{Sm} C^{*}$ phase by application of $15 \mathrm{~V}$ bias and compared with SA samples. Figures 4 and 5 show the memory (static) in SA and UA samples, respectively. Figure 4(a) shows the scattering state before any bias application to the cell. A completely switched bright-dark
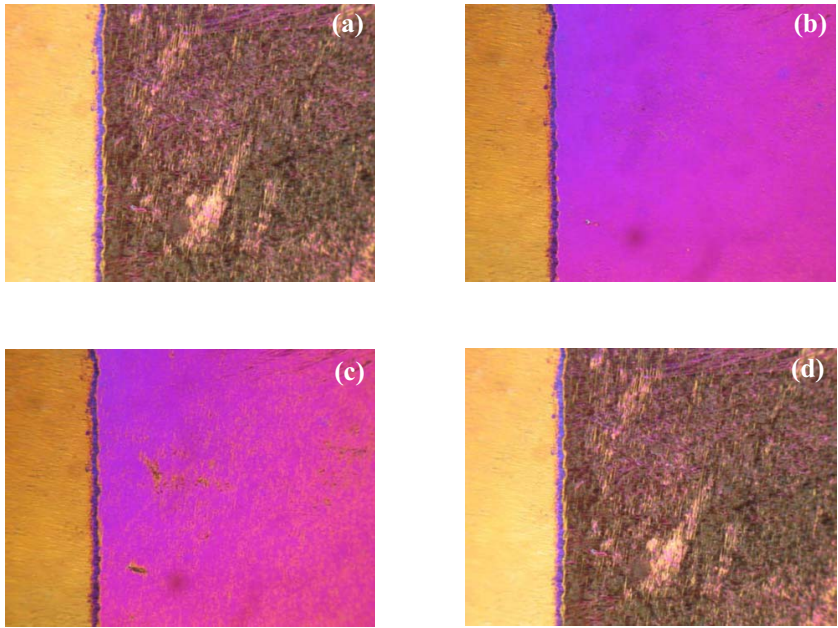

FIG. 5. (Color online) Optical micrographs of 3.5- $\mu$ m UA cells at room temperature at (a) $0 \mathrm{~V}$, (b) $15 \mathrm{~V}$ bias, (c) $30 \mathrm{~min}$ after removal of bias, and (d) again $0 \mathrm{~V}$.

state was achieved on the application of $15 \mathrm{~V}$ bias [Fig. 4(b)]. Figure 4(c) clearly shows that the sample cell has switched back immediately to the original scattering state as the bias was removed. The degradation in memory is due to the strong depolarization and ionic charges, which was also confirmed from Fig. 2. The last (scattering) state [Fig. 4(d)] has been obtained after around $10 \mathrm{~min}$ of the removal of bias. On the contrary the last state has been retained for several hours in UA samples. In Fig. 5(a) the state before the application of bias-i.e., the scattering state-has been shown. A bright-dark state was exhibited when $15 \mathrm{~V}$ bias was applied to the UA samples [Fig. 5(b)]. Figure 5(c) [after $30 \mathrm{~min}$ of the removal of bias] shows that memory was retained even after the removal of bias unlike the SA samples. The last memory state was retained even after a time gap of $90 \mathrm{~h}$ (not shown in the figure) when the bias was removed. This observation of long-lasting static memory ensures the absence of a depolarization field because if this field had been present, the last memory state would not have retained a state like SA samples. So it can be said that the depolarization field was almost absent in UA samples. The absence of a depolarization field can also be confirmed by the fact that the original state did not appear even after shortening of the sample cell. The memory state in the UA sample was switched back forcibly to the original state (scattering state) on the application of a sinusoidal field of low amplitude $(1 \mathrm{~V})$ and high frequency $(30-50 \mathrm{~Hz})$, which is shown in Fig. 5(d). One can say that the molecules of DHFLC material have been clamped onto one side due to electrically induced inertia [38]. In this way, long-lasting memory in UA samples was observed which is due to (i) perfect alignment, (ii) absence of a depolarization field, (iii) absence of ionic charges, and (iv) better stabilization of the helix.

\section{CONCLUSIONS}

A wide range memory effect has been observed in $\mathrm{DH}-$ FLC material without any surface treatment to the cell sur- 
faces. The comparison of this memory effect has been done with strongly anchored DHFLC sample cells. It has been proposed that an ultraweak surface anchoring leads to a lower saturation voltage and steepness in the electro-optical properties of FLCs. A long-lasting memory has been observed in sample cells in which no surface treatment was given. These observations open new ways to understand the effect of surface anchoring on the electro-optical properties of FLC materials and the interaction of FLC molecules with the surface of cell substrates. The underlying concept of achieving the best memory effect can be applied to fabricate paperlike displays because bistable LCDs do not require an electric power for holding an image. Furthermore, the possibility of an ultraweak anchoring provides ideas to pursue future (or new) research-e.g., surface switching and other areas-which will lead to new applications.

\section{ACKNOWLEDGMENTS}

The authors sincerely thank Dr. Vikram Kumar, Director, National Physical Laboratory, for continuous encouragement and interest in this work. We sincerely thank Dr. S. S. Bawa for useful discussions. The authors (J.P. and S.K.) are thankful to CSIR, New Delhi, for financial assistance.
[1] H. Fukuro and S. Kobayashi, Mol. Cryst. Liq. Cryst. 163, 157 (1988).

[2] H. Yokoyama and H. A. van Sprang, J. Appl. Phys. 57, 4520 (1985).

[3] D. S. Seo, T. Oh-ide, and S. Kobayashi, Mol. Cryst. Liq. Cryst. Sci. Technol., Sect. A 214, 97 (1992).

[4] D. S. Seo, K. Muroi, T. Isogami, H. Matsuda, and S. Kobayashi, Jpn. J. Appl. Phys., Part 1 31, 2165 (1992).

[5] S. S. Bawa, A. M. Biradar, K. Saxena, and S. Chandra, Appl. Phys. Lett. 57, 1398 (1990).

[6] I. Dozov, M. Nobili, and G. Durand, Appl. Phys. Lett. 70, 1179 (1996).

[7] H. Yokoyama, Mol. Cryst. Liq. Cryst. 165, 265 (1988).

[8] S. Faetti, Phys. Rev. A 36, 408 (1987).

[9] G. P. Bryan-Brown, E. L. Wood, and I. C. Sage, Nature (London) 399, 338 (1999).

[10] Y. Iimura, N. Kobayashi, and S. Kobayashi, Jpn. J. Appl. Phys., Part 2 33, L434 (1994).

[11] T. Akhane, H. Kaneko, and M. Kimura, Jpn. J. Appl. Phys., Part 1 35, 4434 (1996).

[12] T. Oh-ide, S. Kuniyasu, and S. Kobayashi, Mol. Cryst. Liq. Cryst. 164, 91 (1988).

[13] T. Uchida, M. Hirano, and H. Sakai, Liq. Cryst. 5, 1127 (1989).

[14] A. Murauski, V. Chigrinov, A. Muravsky, Fion Sze-Yan Yeung, J. Ho, and H. S. Kwok, Phys. Rev. E 71, 061707 (2005).

[15] Y. Toko and T. Akhane, Mol. Cryst. Liq. Cryst. Sci. Technol., Sect. A 368, 469 (2001).

[16] M. Nespoulous, C. Blanc, and M. Nobili, J. Appl. Phys. 102, 073519 (2007).

[17] E. P. Haridas, S. S. Bawa, A. M. Biradar, and S. Chandra, Jpn. J. Appl. Phys., Part 1 34, 3602 (1995)

[18] Y. Sato, K. Sato, and T. Uchida, Jpn. J. Appl. Phys., Part 2 31, L579 (1992).

[19] V. P. Vorflusev, H. S. Kitzerow, and V. G. Chigrinov, Appl. Phys. A: Mater. Sci. Process. 64, 615 (1997).

[20] W. S. Kang, H. W. Kim, and J. D. Kim, Liq. Cryst. 29, 583
(2002).

[21] E. Pozhidaev, V. Chigrinov, and X. Li, Jpn. J. Appl. Phys., Part 1 45, 875 (2006).

[22] E. P. Haridas, A. M. Biradar, and G. K. Chadha, Ferroelectrics 211, 9 (1998).

[23] N. A. Clark and S. T. Lagerwall, Ferroelectrics 59, 25 (1984).

[24] L. A. Beresnev, V. G. Chigrinov, D. I. Dergachev, E. P. Poshidaev, J. Funfschilling, and M. Schadt, Liq. Cryst. 5, 1171 (1989).

[25] J. Funfschilling and M. Schadt, J. Appl. Phys. 66, 3877 (1989).

[26] G. B. Cohen, R. Progreb, K. Vinokur, and D. Davidov, Appl. Opt. 36, 455 (1997).

[27] A. M. Biradar, S. Hiller, W. Hasse, E. P. Haridas, S. S. Bawa, and S. Chandra, Liq. Cryst. 20, 641 (1996).

[28] S. S. Bawa, A. M. Biradar, and S. Chandra, Appl. Phys. Lett. 62, 3441 (1993).

[29] K. Miyano, Phys. Rev. Lett. 43, 51 (1979).

[30] J. Nehring, A. R. Kmetz, and T. J. Scheffer, J. Appl. Phys. 47, 850 (1976).

[31] S. S. Bawa, A. M. Biradar, and S. Chandra, Ferroelectrics 76, 69 (1987)

[32] S. S. Bawa, A. M. Biradar, and S. Chandra, Phys. Status Solidi A 102, 829 (1987).

[33] T. C. Chiew and K. H. Yang, Appl. Phys. Lett. 56, 1326 (1990).

[34] K. H. Yang, T. C. Chiew, and S. Osofsky, Appl. Phys. Lett. 55, 125 (1989).

[35] J. Prakash, D. S. Mehta, A. Choudhary, S. Kaur, V. Rathore, and A. M. Biradar, J. Appl. Phys. 103, 044103 (2008).

[36] S. Kaur, A. K. Thakur, R. Chauhan, S. S. Bawa, and A. M. Biradar, J. Appl. Phys. 96, 2547 (2004).

[37] A. Jakli, S. Markscheffel, and A. Saupe, J. Appl. Phys. 79, 1891 (1996).

[38] S. Kaur, A. K. Thakur, S. S. Bawa, and A. M. Biradar, Appl. Phys. Lett. 88, 122905 (2006). 